\title{
Research on global buckling mechanism for submarine pipeline under high temperature and pressure
}

\author{
LIU Wenbin ${ }^{1,2, a, *}$ \\ ${ }^{1}$ Tianjin University, 92 Weijin Rd, Nankai District, Tianjin, China, 300072 \\ ${ }^{2}$ CCCC Tianjin Port Engineering Institute Co., Ltd., 1002 DaGu south Road, Hexi District, Tianjin, \\ China, 300222. \\ a liuwenbin3943@163.com,
}

\begin{abstract}
In order to maintain the flowability of oil, the pipeline is applied high temperature and pressure in working condition. This high temperature and pressure differences makes high stress accumulating in the pipeline, leading to pipeline global buckling. Excessive buckling deformation will cause the pipeline damage and oil spill. This paper reveals the pipeline global buckling mechanism based on the basic mechanics, especially the mechanism of lateral buckling for untrenched pipeline.
\end{abstract}

Keywords: submarine pipeline; lateral global buckling; buckling mechanism

\section{Introduction}

Pipeline systems are economic, safe and efficient systems and transport most of the oil and gas in marine oil and gas engineering ${ }^{[1]}$. The submarine pipeline system typically contains these three parts: gathering line system, exporting line system and risers as Figure 1 shown. This paper focuses the former two structures, gathering and exporting line systems.

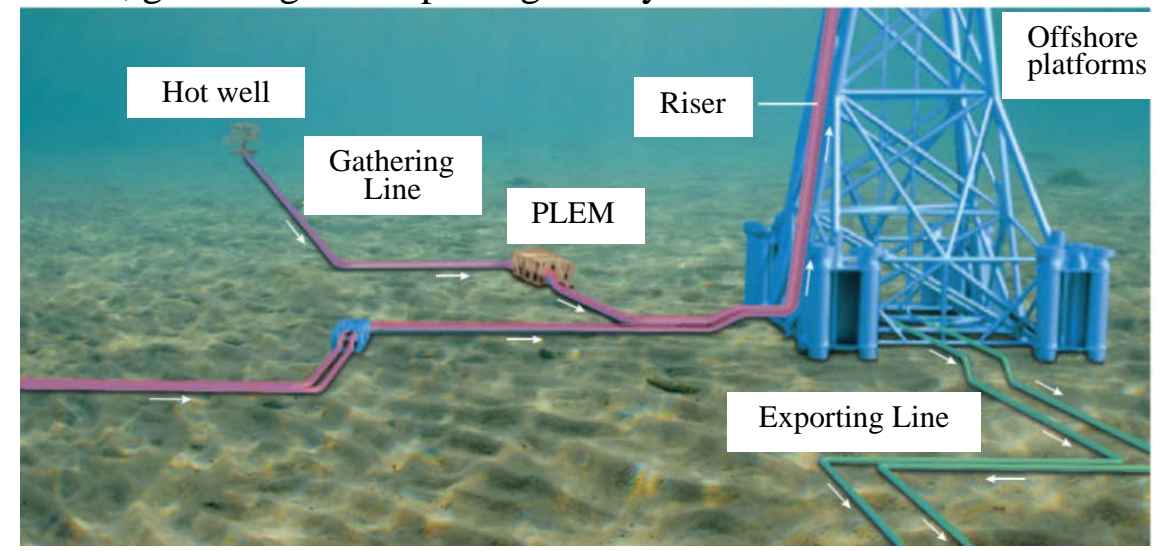

Figure 1 Submarine pipeline system

In order to avoid the solidification of the wax fraction and ease the flow, the submarine pipeline is usually applied high temperature and pressure. This temperature and pressure increases with the increasing water depth and pipeline length ${ }^{[2]}$. In one of the highest temperature and pressure engineering case, the loads reach $177^{\circ} \mathrm{C}$ and $70 \mathrm{MPa}$. The severe working condition and environment challenges the safe design of submarine systems ${ }^{\text {[3]. }}$

The deep-sea pipelines are basically untrenched due to the restriction of high trenching cost and large water depth. These pipeline are restricted by seabed and accumulating large compressive stress under temperature and pressure differences. When the compressive stress reaches a critical value, the 
pipeline occur large and sudden lateral displacement. This large global deformation phenomenon of pipeline is named pipeline global buckling, as Figure 2 shown.

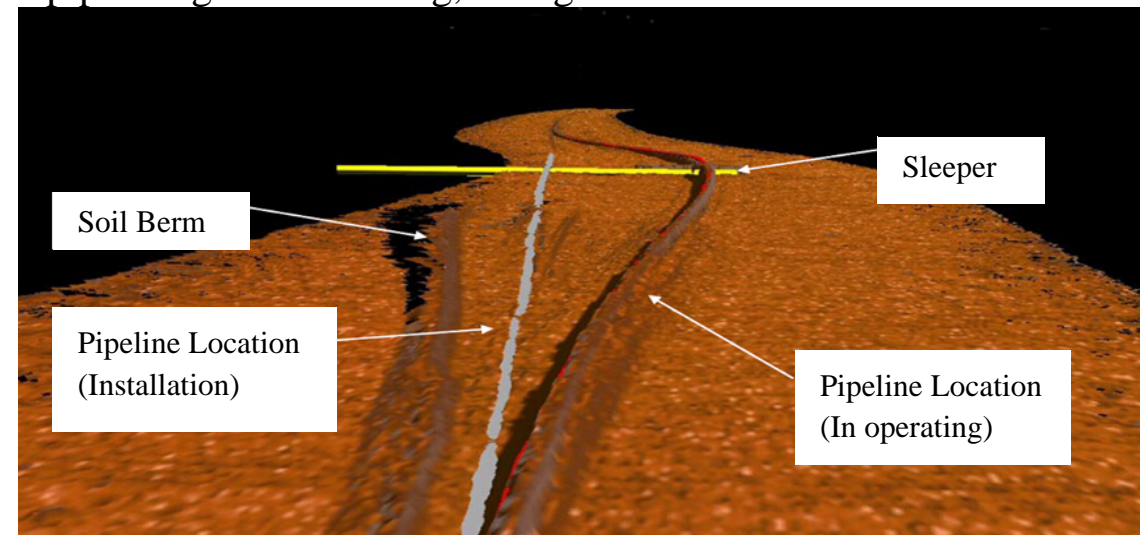

Figure 2 The pipeline lateral global buckling

The pipeline lateral global buckling releases the accumulating stress reducing the risk of material yield, but the large buckling deformation may cause stress concentration harming the pipeline's integrity. Once pipeline is damaged, lots of oil will leak and cost huge financial and environmental lost. In addition, restricting by the water depth, the pipeline repair will be a slow, expensive and challengeable engineering. Thus, predicting the location and deformation of pipeline global buckling is significant for pipeline system design, especially for deep-sea pipelines.

\section{Method}

Pipeline global buckling is similar to stability problems of columns. The column remains balance under the external loads and internal loads. At this time, imposing an extra little force on this column makes column to deviate from the initial position. Then removing this extra force, if this column can restore to its original equilibrium position, we call this system is stable. Otherwise, this system is instable, or can be called global buckling. There are two kinds of instability in practice: bifurcation buckling and snap-through buckling. In order to illustrate the features of the two buckling, the relationship between displacement and load is draw in Figure 3 and Figure 4.

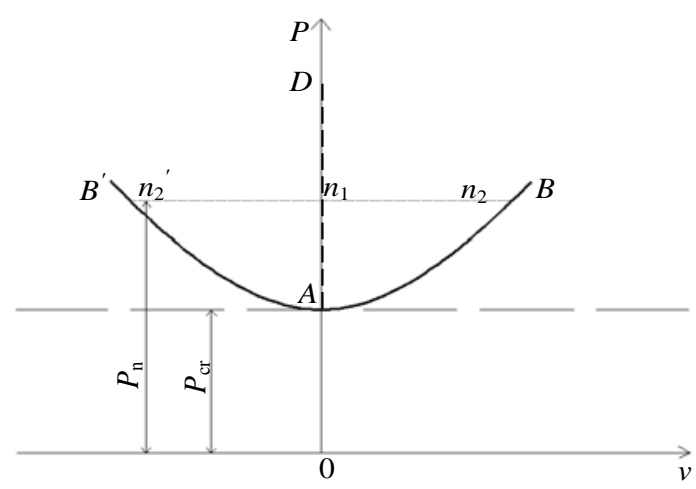

Figure 3 The displacement vs. load for bifurcation buckling

Figure 3 is the relationship between displacement and load for bifurcation buckling. The abscissa is the maximum displacement of instable column, and the ordinate represents the axial compressive force. $P_{\mathrm{cr}}$ is the critical buckling force, and $P_{\mathrm{n}}$ is the axial compressive force applying in the instable structure. The feature of bifurcation buckling is that this kind of buckling has two steady states and one specific stability state changing point $A$. When the axial compressive force is less than this point A, the column will restore to its original equilibrium after the extra force interfering-removing process. When the axial compressive force is reach or larger this point $A$, the column will not recovery stability. Once axial compressive force larger than point A, there exist two opposite stability 
states (curve $\mathrm{AB}$ and curve $\mathrm{AB}$ '). For bifurcation buckling, each axial force $P_{\mathrm{n}}$ has two according displacement $n_{2}$ and $n_{2}$ '.

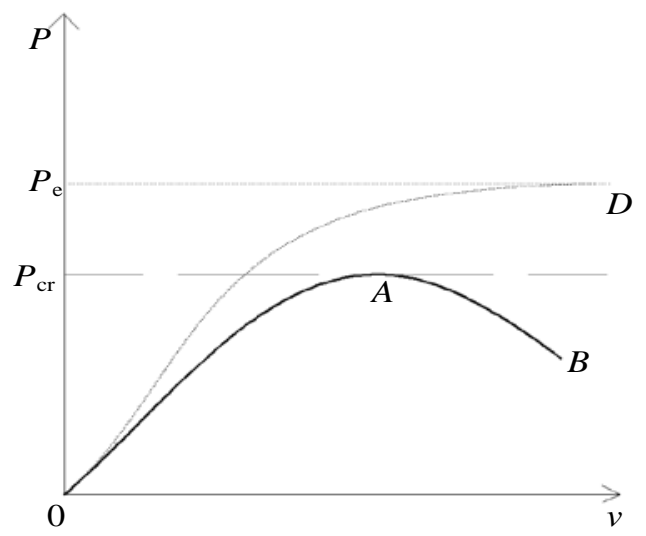

Figure 4 The displacement vs. load for snap-through buckling

Figure 4 is the relationship between displacement and load for snap-through buckling. The abscissa is the maximum displacement of instable column, and the ordinate represents the axial compressive force. $P_{\mathrm{e}}$ is the critical buckling force, and curve OD is the theoretical displacement-load relationship. While in practice, far more before axial force $P$ reaching the value of $P_{\mathrm{e}}$, the outer-side structure section yields and the plastic strain extends to the inside section. The curve $O A B$ is the displacement-load relationship in practice. The feature of snap-through buckling is that the displacement go on increases while the axial force withstanding by structure is decreases after the axial force reach point A. Pipeline global buckling belongs to snap-through buckling.

The deep-sea pipeline is laying directly on the seabed without and trenching processing due to the restriction of difficult deep-water constructing and high engineering cost. The pipeline penetrates into the seabed depending on its self-weight and dynamical effect in installation, as shown in Figure 5. The symbol $z_{p}$ represents the pipeline penetration.

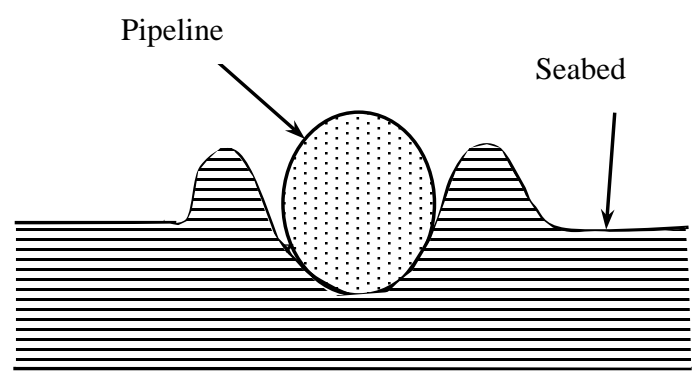

Figure 5 Untrenched pipeline

The pipeline can maintain stability before the load reaches the critical buckling force. High temperature and pressure pipeline accumulates high compressive stress threatening the pipeline stability and occurring global buckling ${ }^{[4]}$. The untrenched pipeline has little lateral soil resistance, and the lateral global buckling is the main buckling form. The limit value of axial compressive stress in pre-buckling pipeline section is called critical buckling force. The high temperature and pressure differences are the direct reason for pipeline global buckling; and the soil resistance leading to stress accumulation is the essential condition. The pipeline global buckling mechanism is that the compressive stress in pipeline cross-section reaches critical buckling force, leading to pipeline instable and occurring large displacement.

\section{Conclusions}

The pipeline global buckling mechanism is that: 1. Due to the thermal expansion and Poisson effect, high temperature and pressure differences generate high compressive stress in pipeline cross-section; 
2. The soil resistance on pipeline restricts the free deformation of pipeline and leads to stress accumulation; 3 . The compressive stress in pipeline cross-section gradually rises, and the stability of pipeline system gradually reduces. When the stress reaches critical buckling force, pipeline becomes instable and occurring large displacement.

\section{Acknowledgments}

This work was financially supported by the Natural Science Foundation of Tianjin (16JCQNJC07200) fund. The authors are grateful for the support provided by Key Laboratory of Port Geotechnical Engineering of Tianjin and Key Laboratory of Port Geotechnical Engineering, Ministry of Communications, PRC.

\section{References}

[1] Ribo S. Latest Progress of Development of Deep Water Offshore Oil and Gas in China[J]. Shipbuilding of China S, 2012, 1.

[2] Konuk I. Expansion of Pipelines under Cyclic Operational Conditions: Formulation of Problem and Development of Solution Algorithm[C]//Proceedings of the 17th International Conference on Offshore Mechanics and Arctic Engineering (OMAE1998). 1998.

[3] Carr M, Bruton D, Leslie D. Lateral buckling and pipeline walking, a challenge for hot pipelines[C]//Offshore Pipeline Technology Conference, Amsterdam. 2003: 1-36.

[4] Liu Hongwen .Mechanics of materials[M]. Beijing: Beijing, 2011. 